\title{
Massive Bleeding from a Small Intestinal Submucosal Tumor-like Arterial Malformation: An Unclassifiable Vascular Lesion Revealed by a Detailed Pathological Evaluation
}

\begin{abstract}
Kazuhiro Ota ${ }^{1}$, Junki Noda ${ }^{1}$, Hiroshi Akutagawa $^{2}$, Noriaki Sugawara ${ }^{1}$, Kei Nakazawa ${ }^{1}$, Shinya Nishida ${ }^{1}$, Yuki Hirata ${ }^{1}$, Satoshi Harada ${ }^{1}$, Wataru Osumi ${ }^{3}$, Yuichi Kojima ${ }^{1}$, Sadaharu Nouda ${ }^{1}$, Toshihisa Takeuchi ${ }^{1}$, Yoshinobu Hirose ${ }^{2}$ and Kazuhide Higuchi ${ }^{1}$
\end{abstract}

\begin{abstract}
:
A 53-year-old woman presented with repeated copious bloody stool. Small bowel capsule endoscopy revealed a submucosal tumor (SMT)-like lesion, with erosion of the surface, in the first third of the small bowel. Balloon-assisted small intestinal endoscopy also revealed a pulsatile SMT-like lesion with an exposed vessel on the surface. This unknown lesion was surgically resected. The histopathological findings of the resected SMT-like lesion showed a dilated artery with thrombosis blockage and recanalization. Since this case could not be classified as any of the small intestinal vascular lesion patterns endoscopically, its classification will require the accumulation of further cases.
\end{abstract}

Key words: obscure gastrointestinal bleeding, capsule endoscopy, small intestinal bleeding

(Intern Med 58: 3521-3523, 2019)

(DOI: 10.2169/internalmedicine.3217-19)

\section{Introduction}

Histopathologically, small intestinal vascular lesions have been classified as angioectasia, Dieulafoy's lesion, and arteriovenous malformation. The Yano-Yamamoto classification is widely used for the endoscopic classification of small intestinal vascular lesions (Table) (1). It is generally agreed that the Yano-Yamamoto classification types $1 \mathrm{a}$ and $1 \mathrm{~b}$ correspond to angioectasia, types $2 \mathrm{a}$ and $2 \mathrm{~b}$ to Dieulafoy's lesion, and type 3 to arteriovenous malformation; however, type 4 does not correspond to a specific lesion. Some types of small intestinal lesions cause massive arterial bleeding, including arterial vascular lesions (Dieulafoy's lesion and arteriovenous malformation), ulcers, and malignant tumors (gastrointestinal stromal tumor and cancer). Small intestinal arterial bleeding often causes recurrent episodes of sudden massive bloody stool and hemorrhagic shock without ab- dominal pain and requires a long-term examination to detect its origin. For these reasons, unidentified and unclassified lesions should be further studied.

We herein report a case of massive bleeding from a small intestinal submucosal tumor (SMT)-like arterial malformation.

\section{Case Report}

A 53-year-old woman presented with recurrent copious bloody stool and hemorrhagic shock. She underwent dynamic contrast-enhanced computed tomography, emergent esophagogastroduodenoscopy, and total colonoscopy at another hospital; however, the bleeding origin could not be identified. Therefore, she was transferred to our hospital for the further investigation of the small intestinal bleeding. Because small bowel capsule endoscopy (PillCam ${ }^{\circledR} \mathrm{SB} 3$; Covidien, Dublin, Ireland) revealed a hemispherical SMT-

${ }^{1}$ Second Department of Internal Medicine, Osaka Medical College, Japan, ${ }^{2}$ Department of Pathology, Osaka Medical College, Japan and ${ }^{3}$ Department of General and Gastroenterological Surgery, Osaka Medical College, Japan

Received: April 19, 2019; Accepted: June 23, 2019; Advance Publication by J-STAGE: August 6, 2019

Correspondence to Dr. Kazuhiro Ota, clash_kaz@yahoo.co.jp 
Table. Endoscopic Classification of Small Intestinal Vascular Lesion (Yano-Yamamoto Classification).

\begin{tabular}{ll}
\hline Type 1a & Puncture erythema $(<1 \mathrm{~mm})$ with or without oozing \\
Type 1b & Patchy erythema $(\mathrm{a}$ few $\mathrm{mm})$ with or without oozing \\
Type 2a & Puncture lesions $(<1 \mathrm{~mm})$ with pulsatile bleeding \\
Type 2b & Pulsatile red protrusion without surrounding venous dilation \\
Type 3 & Pulsatile red protrusion with surrounding venous dilation \\
Type 4 & Other lesions not classified into any of the above categories \\
\hline
\end{tabular}

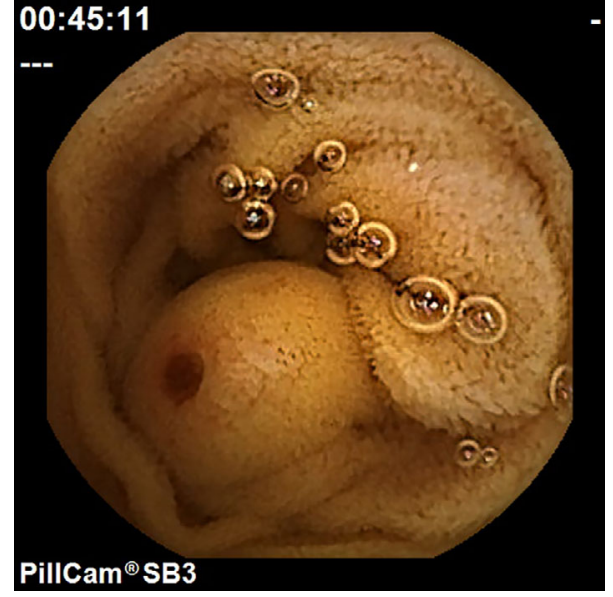

Figure 1. Capsule endoscopic findings (PillCam ${ }^{\circledR}$ SB3; Covidien, Dublin, Ireland) revealed a hemispherical submucosal tumor-like lesion with erosion at the surface in the first third of the small bowel.

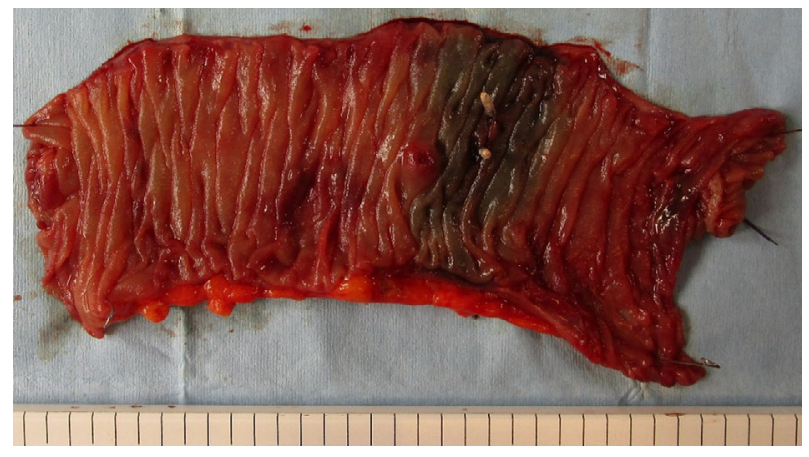

Figure 3. Macroscopic findings of the surgically resected specimen. Note the submucosal tumor-like lesion with erosion at the anal side of the markings with clipping and inking.

like lesion with erosion of the surface in the first third of the small bowel, the lesion was identified as the bleeding origin (Fig. 1, Supplementary material). Transoral doubleballoon enteroscopy also revealed a pulsatile hemispherical SMT-like lesion with an exposed vessel on the surface, not accompanied by surrounding venous dilatation (Fig. 2).

The lesion was classified as type 4 (unclassifiable) in the Yano-Yamamoto classification (1). However, although gastrointestinal stromal tumor and arteriovenous malformation were included as the differential diagnosis of this lesion, it could not be definitively diagnosed by endoscopic findings and previous studies alone. Furthermore, because the com-

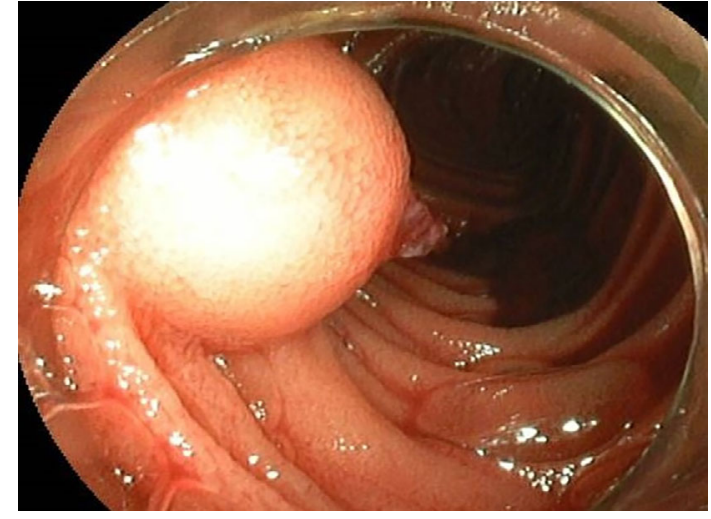

Figure 2. Transoral double-balloon enteroscopy also revealed a pulsatile hemispherical submucosal tumor-like lesion with an exposed vessel at the surface.

pleteness of endoscopic hemostasis for this unknown lesion remained uncertain, it was surgically resected (Fig. 3). The histopathological findings of the resected SMT-like lesion showed a dilated artery with thrombosis blockage and recanalization. In a high-magnification Elastica van Giesonstained image, the elastic lamina was confirmed to have no venous component (Fig. 4).

\section{Discussion}

In the present case, dynamic contrast-enhanced computed tomography did not reveal the bleeding origin; however, small bowel capsule endoscopy effectively detected the origin and location of bleeding to guide the approach route of balloon-assisted enteroscopy, which did not achieve hemostasis but effectively marked the lesion for surgical resection. The immediate diagnosis of the occult gastrointestinal bleeding based on an efficient strategy is necessary for early hemostasis (2). When deciding on the treatment strategy for bleeding lesions with uncertain endoscopic diagnosis, endoscopic hemostasis should be carefully evaluated (3).

Several previous case reports have been conducted on small intestinal vascular malformations causing obscure gastrointestinal bleeding. Liao et al. reported a vascular malformation in the small intestine that was an SMT-like lesion with erosion at the surface, as detected endoscopically. Their case report's common pathological point is similar to that of our case: thrombosis with thick-walled blood vessels detected histologically (4). Sai et al. considered that an ele- 

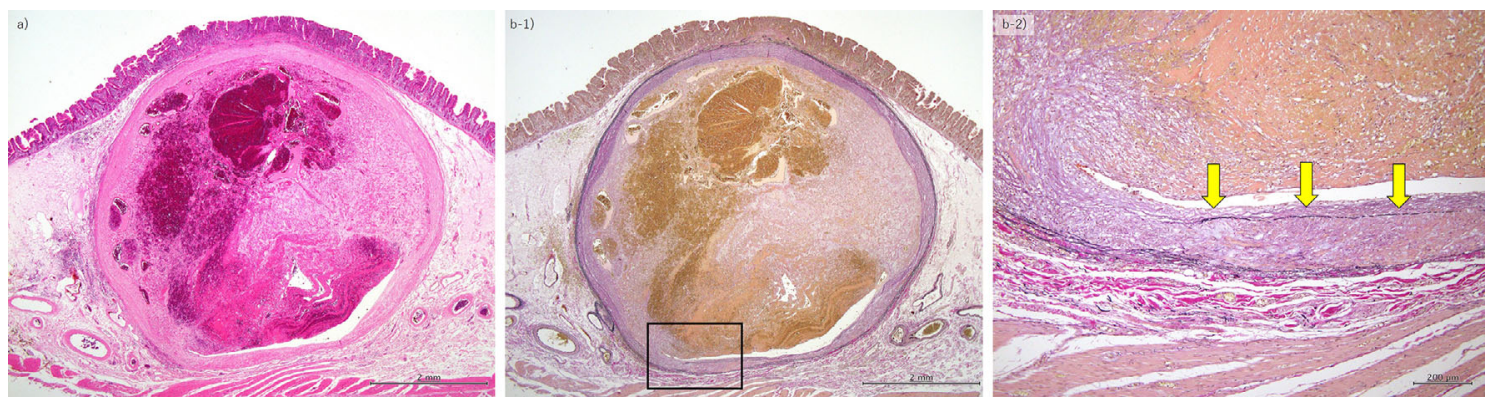

Figure 4. Microscopic findings showing a dilated artery with thrombosis blockage and recanalization, rather than a tumor. In the high-magnification image on Elastica van Gieson staining, the elastic lamina was confirmed to have no venous component. a) Hematoxylin and Eosin staining. b) Elastica van Gieson staining. b-1) low-magnification image: the black square area shows the high magnification image shown below; b-2) high-magnification image: the yellow arrows show the elastic lamina.

vated lesion was formed due to thrombosis near the Dieulafoy's lesion (5). Endoscopically, type 2 lesions in the YanoYamamoto classification may progress to an SMT-like lesion due to thrombosis and fibrosis. Onogi et al. reported a case of arteriovenous malformation that formed an SMT-like finding accompanied by a large arterial vessel with inner thrombosis (6). In the present case, the blood vessel was considered to have been dilated because of arterial thrombosis of unknown etiology, creating an SMT-like lesion endoscopically.

Although small intestinal vascular lesions have been classified into three pathological patterns (angioectasia, Dieulafoy's lesion, and arteriovenous malformation), this case might be one of Dieulafoy's lesion, as there was no venous component. There have been several case reports of submucosal aneurysm of the small intestine, and the pathologic findings might be the same as in the present case (7-9). The formation of a hemispherical submucosal tumor-like lesion endoscopically may have been due to the thrombosis in the arterial vessel causing focal arterial dilation. Generally, it has been reported that Dieulafoy's lesion often appeared endoscopically as a type 2 lesion in the Yano-Yamamoto classification (1). Because a hemispherical SMT-like lesion derived from Dieulafoy's lesion was seen in the present case as well as in others reported in the literature, the endoscopic classification of small intestinal vascular lesions might require reconsideration. Imamura et al. referred to such a lesion as "Dieulafoy's vascular malformation" based on a histopathological assessment (10). To establish a new classification of hemispherical SMT-like protrusions with an exposed vessel, further cases will need to be accumulated.

The authors state that they have no Conflict of Interest (COI).

\section{References}

1. Yano T, Yamamoto H, Sunada K, et al. Endoscopic classification of vascular lesions of the small intestine (with videos). Gastrointest Endosc 67: 169-172, 2008.

2. Yamamoto $\mathrm{H}$, Ogata $\mathrm{H}$, Matsumoto $\mathrm{T}$, et al. Clinical practice guideline for enteroscopy. Dig Endosc 29: 519-546, 2017.

3. Ota K, Tatsumi Y, Matsui M, et al. Case of massive hematemesis by secondary aortic duodenal fistula 8 years after abdominal aortic aneurysm repair. Geriatr Gerontol Int 17: 1234-1235, 2017.

4. Liao Z, Gao R, Li ZS. Vascular malformation of the small intestine. Endoscopy 39 (Suppl): E319, 2007.

5. Sai Prasad TR, Lim KH, Yap TL. Bleeding jejunal Dieulafoy pseudopolyp: capsule endoscopic detection and laparoscopicassisted resection. J Laparoendosc Adv Surg Tech A 17: 509-512, 2007.

6. Onogi F, Araki H, Kimura YT, et al. A case of anteriovenous malformation of the jejunum diagnosed by double-balloon enteroscopy and contrast-enhanced computed tomography in abdomen. Gastroenterol Endosc 51: 1301-1308, 2009 (in Japanese, Abstract in English).

7. Chiba H, Endo K, Fujishima F, et al. A case of a ruptured submucosal aneurysm of the small intestine identified using doubleballoon enteroscopy. Clin J Gastroenterol 9: 49-54, 2016.

8. Naito M, Sato B, Kida A, et al. Ruptured submucosal aneurysm in the jejunum resected by urgent laparoscopic surgery. Jpn J Gastroenterol Surg 49: 905-910, 2016 (in Japanese).

9. Tadano T, Noguchi K, Endo K, et al. A case of ruptured submucosal aneurysm of the small intestine observed by double-balloon enteroscopy. Gastroenterol Endsc 55: 1827-1834, 2013 (in Japanese, Abstract in English).

10. Imamura N, Maehara N, Hotokezawa M, Chijiiwa K. A case of Dieulafoy's vascular malformation of the small intestine resected with the aid of preoperative coiling. Nihon Rinsho Geka Gakkai Zasshi (J Japan Surg Assoc) 68: 2517-2521, 2007 (in Japanese, Abstract in English).

The Internal Medicine is an Open Access journal distributed under the Creative Commons Attribution-NonCommercial-NoDerivatives 4.0 International License. To view the details of this license, please visit (https://creativecommons.org/licenses/ by-nc-nd/4.0/).

(C) 2019 The Japanese Society of Internal Medicine Intern Med 58: 3521-3523, 2019 